\title{
Comparison of unilateral pedicle screw fixation and interbody fusion with PEEK cage vs. standalone expandable fusion cage for the treatment of unilateral lumbar disc herniation
}

Jinlei Zhang'1, Aixing Pan², Li Zhou ${ }^{1}$, Jingyi Yu¹, Xiao Zhang ${ }^{1}$

\author{
${ }^{1}$ Department of Orthopaedics, Zhoukou City Central Hospital, The Affiliated Hospital \\ of Xinxiang Medical College, Zhoukou Shi, China \\ 2Department of Orthopaedics, Beijing Chao-Yang Hospital, Capital Medical University, \\ Beijing, China
}

Submitted: 23 October 2017

Accepted: 17 February 2018

Arch Med Sci 2018; 14, 6: 1432-1438

DOI: https://doi.org/10.5114/aoms.2018.74890

Copyright $\odot 2018$ Termedia \& Banach

\section{Abstract}

Introduction: This study was conducted to compare the clinical effects of unilateral pedicle screw fixation and interbody fusion with PEEK cage (UPSFC) and standalone expandable fusion cage (SAEFC) on unilateral lumbar disc herniation.

Material and methods: From September 2011 to July 2014, a respective investigation was performed on 130 lumbar disc herniation patients treated with SAEFC or UPSFC. The hospital stay, operating time, blood loss, Japanese orthopaedic association scores (JOA), and visual analogue score (VAS) in the two groups were compared using Student's $t$-test.

Results: The average of follow-up time was $25.6 \pm 6.4$ and $25.2 \pm 5.8$ months, respectively. No significant difference in the postoperative hospitalizsation, intraoperative blood loss, operative time, and postoperative fusion rate was detected between the two groups. VAS score in the UPSFC group was significantly lower than in the SAEFC group at 6 and 12 months after operation $(p=0.014, p=0.004)$. X-ray images indicated that the subsidence rate was $8.1 \%(5 / 62)$ in the SAEFC group, while no subsidence was detected in UPSFC group 12 month after operation.

Conclusions: Both SAEFC and UPSFC are effective techniques. UPSFC may be a better choice for patients with lumbar disc herniation and unilateral limb symptoms of nerve root in view of the advantages of better low back pain relief and low subsidence rate.

Key words: lumbar disc herniation, unilateral pedicle screw fixation and interbody fusion with PEEK cage, standalone expandable fusion cage, Peek cage.

\section{Introduction}

Lumbar disc herniation is a common condition causing back pain and/ or leg pain/numbness, but its occurrence, diagnosis, and clinical outcomes after conservative treatment with medication have been poorly documented [1]. Recurrent lumbar disc herniation is defined as disc herniation at previously operated disc level in patients who experienced a pain-free interval of at least 6 months after surgery [2]. Recurrent disc herniation is a significant problem because scar formation may lead to increased mor-

\author{
Corresponding author: \\ Dr. Jinlei Zhang \\ Department \\ of Orthopaedics \\ Zhoukou City \\ Central Hospital \\ The Affiliated Hospital \\ of Xinxiang \\ Medical College \\ 26 Renmin Road \\ Zhoukou City 46600 \\ Henan Province, China \\ Phone/fax: \\ +8618135750501 \\ E-mail: iqi001@163.com
}


bidity after traditional posterior reoperation [3]. Furthermore, persistent low back pain or recurrent sciatica may develop in some cases after repeated surgery, and it is important to consider the possibility of iatrogenic instability during surgery on the lumbar spine. Therefore, a stable and effective expectant treatment to relieve the neurothlipsis for patients has become necessary.

Lumbar fusion has been proven to have an excellent clinical effect after more than 100 years of development since Albee and Hibbs first used it in 1911 , and has become one of the commonly operative methods for treating lumbar degenerative diseases, which includes posterolateral fusion and interbody fusions $[4,5]$. Interbody fusion cage, which is a widely accepted approach to stabilise the spinal segment of surgery, is utilised to perform lumbar interbody fusion and assist in the recovery of the height between the intervertebral space and the foramen after neurological decompression. However, most fusion cages designed early are required for assistance with pedicle internal fixation to enhance the biological stability of the cage because these fusion cages had defects such as subsidence and displacement, and the intervertebral height lost cannot be used alone [6].

Generally, bilateral pedicle screw fixation is accepted as a standard procedure in lumbar interbody fusion. And bilateral pedicle screw is characterised by high biomechanical stability and several clinical advantages such as correction of deformities, maintenance of the height of the intervertebral disc, improvement of interbody fusion, and acceleration of the recovery process after spine surgery [7]. However, a patient with more implants experiences more extensive dissection, greater blood loss, longer duration of operation, and higher risk of implant-related complications. Also, it may mean greater medical costs, thus increasing the economic burden to patients living in poor areas. To reduce these problems some researchers have performed unilateral pedicle screw fixation, which has obtained satisfactory clinical outcomes [8, 9].

Compared with bilateral pedicle screw fixation, unilateral pedicle screw fixation is characterised by a single, small, paramedian muscle-splitting exposure that results in reduced blood loss and hospital stay $[10,11]$. In addition, some studies have shown that a greater number of implants and rigid fixation can cause more clinically adverse effects, including reduction of the fusion rate and adjacent segment degeneration $[9,12,13]$. However, recent reports indicated that there was no significant difference between bilateral and unilateral pedicle screw fixation in the treatment of single and double segmental lumbar degenerative disease.
Also, the fusional rate and complication induced by unilateral pedicle screw fixation is the same as for bilateral pedicle screw fixation $[8,14,15]$.

In order to improve adverse factors induced by pedicle screw fixation, a standalone expandable fusion cage (SAEFC) has been developed, and this fusion cage utilises the principle of leverage to boost safety and clinical success by providing improved stability, preservation of lordosis, and resistance to subsidence and migration, which has been found in earlier interbody cage designs [16].

The purpose of this report is to compare the clinical effects of SAEFC and unilateral pedicle screw fixation and interbody fusion with PEEK cage (UPSFC) in the treatment of lumbar disc herniation. We provide a method and detail information for operation of lumbar disc herniation in the hope that this approach will assist surgeons in selecting a better way of curing the disease.

\section{Material and methods}

\section{Patients and inclusion criteria}

From September 2011 to July 2014 a respective investigation was performed on 130 lumbar disc herniation patients who were treated with SAEFC or UPSFC.

Inclusion criteria were as follows: 1) unilateral disc herniation of single segment diagnosed by clinical symptoms and signs and preoperative X-ray imaging, computed tomography (CT), and magnetic resonance imaging (MRI) examinations, 2) central or subarticular disc herniation, 3) presence of radicular symptoms on the unilateral lower limb, 4) failure of conservative treatment for at least 6 months.

Exclusion criteria were as follows: 1) lumbar instability, 2) extreme lateral or foraminal lumbar disc herniation, 3) osteoporosis, 4) recurrence or secondary surgery, 5) insufficiency of imaging data, 6) follow-up period less than 12 months, 7) other disease such trauma, infection, and tumour, 8) developmental spinal stenosis or lumbar spondylolisthesis.

The patients were assigned into either the SAFEC of UPSFC group according to the decision of therapeutic regimen made by the chief surgeons; 62 patients received SAEFC and 68 patients received UPSFC.

\section{Surgical procedure}

All patients in this surgery underwent intravenous composite anaesthesia in the prone position. Posterior lumbar interbody fusion (PLIF) were performed on all patients.

In the SAEFC group, "C"-arm X-ray was used to locate the intervertebral space accurately. After proper skin preparation, a nearly $6-\mathrm{cm}$ skin inci- 
sion was made. The skin and subcutaneous tissue were cut one by one, and the vertebral bodies on both sides of the vertebral plate were exposed. After the exposure of the upper and lower articular processes, the ligamentum flavum was excised. The superior border of the vertebral plate located at the lower lamina, inferior margin of the vertebral plate located at upper lamina, and lateral margin of the zygapophysial joints were removed from the operated segment. Decompression of the lateral recess was conducted, and the nerve root was relieved following the cleaning of the nerve root export. The intervertebral disc and cartilage were removed while the integrity of the lamina terminalis was well protected. Then the dural sac and nerve root were retracted to one side, and the bone particles taken off at the time of decompression were placed in the intervertebral disc space. Expandable titanium cage filled with autogenous bone was implanted deeply enough into the intervertebral space. The cage was expanded by stretching screw with the assistance of a long rod screwdriver.

The decompression of UPSFC was the same as SAEFC after unilateral pedicle screw fixation, and autograft and allograft bone grafts were placed in the intervertebral disc space. A polyetheretherketone cage (PEEK cage) was expanded by a stretching screw with the assistance of a long rod screwdriver. The cage was implanted deeply enough below $5 \mathrm{~mm}$ at the vertebral rear.

A drainage tube was routinely placed postoperatively and removed $24-48 \mathrm{~h}$ later. Routine administration of antibiotics was used for $48 \mathrm{~h}$. The utmost care was taken to avoid neural damage.

\section{Follow-up and outcome measurement}

All patients were followed up regularly at 1 week, 3 months, 6 months, and 12 months. And 1 year after operation, patients who accepted operation were rechecked once a year. The follow-up visit included radiographic evaluation and objective evaluation of clinical curative effect. Criteria to judge the bone graft fusion were as follows [17]: 1) determination of intervertebral space passed through by bone trabecula, 2) less than $4^{\circ}$ in angular variation during fusion segments detected by X-ray, 3) no obvious translucency detected at the interface between the cage and the centrum.

Blood loss, operation time, and hospital stay were recorded for each patient. Japanese orthopaedic association scores (JOA) was used to evaluate the improvement of clinical function. The VAS pain score was measured by asking the patient to locate the severity of the pain on a horizontal line and score it on a scale of 0 to 10 , with 0 representing no pain and 10 representing the most severe pain.
The follow-up and postoperative $\mathrm{X}$-ray films of the patients were compared. A cage shift into the tail end of the vertebral body more than $3 \mathrm{~mm}$ was defined as subsidence. A distance between the posterior edge of the fusion device and the posterior margin of the tail vertebra of over $3 \mathrm{~mm}$ was defined as a shift

\section{Statistical analysis}

Data analyses were performed by using SPSS software, version 23.0 (SPSS Inc., Chicago, IL). Quantitative data were expressed as means \pm standard deviations (SD). Qualitative data were expressed as number and percentage. Independent samples $t$-test and Mann Whitney $U$ test were used for comparisons of quantitative data between two groups. Repeated ANOVA measures were used for comparisons of different scores at each time point. A $p$-value of less than 0.05 was considered as statistically significant.

\section{Results}

\section{Baseline characteristics}

The demographic characteristics of patients in the two groups are shown in Table I. There was no statistically significant difference between the groups in terms of age, gender, segments of operation, postoperative hospitalisation, intraoperative blood loss, and follow-up time. Longer operative time was detected in the UPSFC group than in the SAEFC group $(64.2 \pm 5.77$ vs. $68.7 \pm 7.64 \mathrm{~min}$, $p<0.05)$.

\section{Surgical outcomes}

All cages were successfully implanted by a single attempt. A case of intervertebral infection was detected in the SAEFC group, and the cage was removed and immobilised into the intervertebral space by bilateral internal fixation. No significant difference was detected between the two groups in terms of postoperative hospitalisation, blood loss, operation time, and fusion rate. The surgical outcomes of the two groups are shown in Table II.

\section{JOA and VAS score}

All patients completed the self-reported questionnaires as instructed. The JOA scores at follow-up period of $1,3,6$, and 12 months were significantly increased compared to preoperative scores $(p<$ 0.01 ). No significant different was found in pointin-time JOA scores between the SAEFC and UPSFC groups. The low back pain VAS score was significantly decreased in both groups when compared with preoperative VAS score. And the low back pain VAS score in the SAEFC group was significantly higher than that in the UPSFC group at post-opera- 
Table I. Demographic information of lumbar disc herniation patients in the two groups

\begin{tabular}{|c|c|c|c|}
\hline Parameters & SAEFC group $(n=62)$ & UPSFC group $(n=68)$ & $P$-value \\
\hline Gender (male/female) & $36 / 26$ & $40 / 28$ & 0.681 \\
\hline Age [years] & $50.9 \pm 7.3$ & $49.3 \pm 6.3$ & 0.195 \\
\hline \multicolumn{4}{|c|}{ Concomitant diseases, $n(\%)$ : } \\
\hline Diabetes & $6(9.7)$ & $10(14.7)$ & 0.383 \\
\hline Obesity & $5(8.1)$ & $4(5.9)$ & 0.736 \\
\hline Hypertension & $15(24.2)$ & $20(29.4)$ & 0.503 \\
\hline Hypercholestermia & $9(14.5)$ & $5(7.4)$ & 0.188 \\
\hline \multicolumn{4}{|l|}{ Segments of operation: } \\
\hline L3-L4 & 14 & 12 & \\
\hline L4-L5 & 32 & 40 & 0.681 \\
\hline L5-S1 & 16 & 16 & \\
\hline Follow-up [months] & $25.6 \pm 6.4$ & $25.2 \pm 5.8$ & 0.728 \\
\hline
\end{tabular}

Table II. Surgical outcomes of SAEFC and UPSFC group

\begin{tabular}{|lccc|}
\hline Parameters & SAEFC group $(n=62)$ & UPSFC group $(n=68)$ & $P$-value \\
\hline Postoperative hospitalisation [days] & $10.0 \pm 1.43$ & $9.8 \pm 1.61$ & 0.51 \\
\hline Blood loss [ml] & $324.0 \pm 22.4$ & $339.4 \pm 62.8$ & 0.07 \\
\hline Operation time [min] & $64.2 \pm 5.77$ & $68.7 \pm 7.64$ & 0.06 \\
\hline Fusion rate & $91.9 \%(57 / 62)$ & $94.1 \%(64 / 68)$ & 0.74 \\
\hline
\end{tabular}

Table III. Comparisons between SAEFC group and UPSFC group regarding JOA and VAS scores

\begin{tabular}{|lcccccc|}
\hline Parameter & Group & Baseline & 1 months & 3 months & 6 months & 12 months \\
\hline JOA & SAEFC & $17.23 \pm 1.273$ & $21.32 \pm 1.36^{\mathrm{a}}$ & $24.23 \pm 1.60^{\mathrm{a}}$ & $26.47 \pm 1.11^{\mathrm{a}}$ & $27.32 \pm 1.27^{\mathrm{a}}$ \\
\cline { 2 - 7 } & UPSFC & $16.88 \pm 1.42$ & $21.15 \pm 1.61^{\mathrm{a}}$ & $23.74 \pm 1.74^{\mathrm{a}}$ & $26.13 \pm 1.32^{\mathrm{a}}$ & $26.91 \pm 1.37^{\mathrm{a}}$ \\
\hline \multirow{2}{*}{ VAS (back) } & SAEFC & $5.50 \pm 1.566$ & $4.26 \pm 2.38^{\mathrm{a}}$ & $3.13 \pm 2.28^{\mathrm{a}}$ & $2.63 \pm 1.37^{\mathrm{a}}$ & $2.61 \pm 1.37^{\mathrm{a}}$ \\
\cline { 2 - 7 } & UPSFC & $5.90 \pm 2.14$ & $3.91 \pm 1.66^{\mathrm{a}}$ & $2.62 \pm 1.74^{\mathrm{a}}$ & $2.03 \pm 1.37^{\mathrm{a}, \mathrm{b}}$ & $1.88 \pm 1.44^{\mathrm{a}, \mathrm{b}}$ \\
\hline VAS (leg) & SAEFC & $6.00 \pm 2.40$ & $2.61 \pm 1.44^{\mathrm{a}}$ & $1.97 \pm 1.34^{\mathrm{a}}$ & $2.10 \pm 1.67^{\mathrm{a}}$ & $1.95 \pm 1.61^{\mathrm{a}}$ \\
\cline { 2 - 7 } & UPSFC & $6.03 \pm 2.02$ & $2.50 \pm 1.30^{\mathrm{a}}$ & $2.13 \pm 1.63^{\mathrm{a}}$ & $1.87 \pm 1.34^{\mathrm{a}}$ & $1.82 \pm 1.34^{\mathrm{a}}$ \\
\hline
\end{tabular}

aPost-operative vs. baseline scores, $p<0.05$; ${ }^{b}$ UPSFC vs. SAEFC, $p<0.05$.

tive 6 and 12 months $(p<0.05)$. The point-in-time VAS score of melosalgia in both groups was significantly decreased in comparison with pre-operation. No significant difference was found in point-in-time VAS score of melosalgia between the SAEFC and UPSFC groups. Detailed information about JOA and VAS scores is shown in Table III.

\section{Radiological results}

Twelve months after operation, radiological bony fusion was achieved in 57 (91.9\%) patients from the SAEFC group and in 64 (94.1\%) patients from the UPSFC group. There was no significant difference in bony fusion between the two groups $(p=0.736, p>0.05)$. Compared with preoperative $X$-ray image, the postoperative image indicated that the of subsidence ratio of the SAEFC group was $8.1 \%(5 / 62)$ and the shifting ratio of the SAEFC group was $0 \%(0 / 62)$. No subsidence and shifting was observed in the UPSFC group. Typical pre- and post-operative images of patients who received SAEFC or UPSFC are shown in Figures 1 and 2.

\section{Complications}

One case in each group had a superficial infection. The wound recovered after dismantling some sutures and wound dressing. In the SAEFC group, one case of spinal dural rupture occurred during operation, which is related to intraoperative adhesion and careless operation. It was repaired during operation without injury of the nerve root and recovered well after operation. 

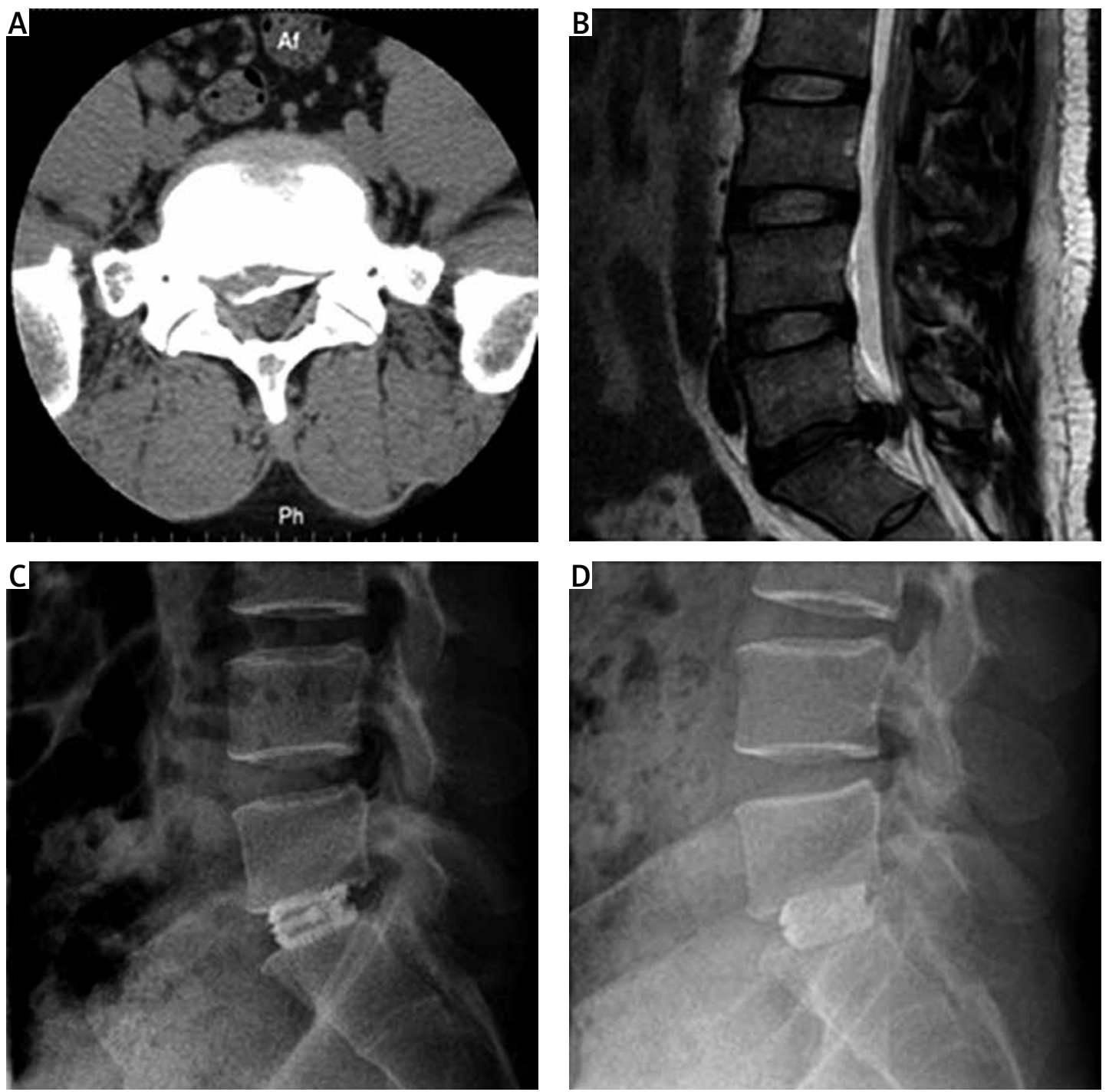

Figure 1. Pre- and post-operative images of a patient who received SAEFC. A - The preoperative image of CT scan for central disc herniation, which was accompanied by calcification. B - Preoperative T2 WI MRI scanning for protrusion of intervertebral disc at L5/S1. C - X-ray image of lateral projection detected on 1 week after operation. D - X-ray image of lateral projection detected 1 year after operation

\section{Discussion}

Previous studies have demonstrated that both SAEFC and UPSFC can achieve satisfactory effects in the treatment of lumbar disc herniation $[14,16,18$, 19]. However, there has been no consensus regarding the optimal surgical method in the treatment of lumbar disc herniation because they have similar operative routes and clinical outcomes. To our knowledge, there has been no previous study comparing the clinical effects of SAEFC and UPSFC on unilateral lumbar disc herniation Therefore, in this study, we compared the clinical efficacy of these two methods and found that VAS score in the UPSFC group was significantly lower than in the SAEFC group at 6 and 12 months after operation. X-ray images indicate that the subsidence rate was $8.1 \%$ in the SAEFC group, while no subsidence was detected in the UPSFC group 12 month after operation.
In our study, the incidence of subsidence was $8.1 \%$ and $0 \%$ in the SAEFC group and UPSFC group, respectively, while the fusion rate was $91.9 \%$ and $94.1 \%$ in the SAEFC group and UPSFC group, respectively. These findings are consistent with previous studies [18, 20-23]. Park et al. found that the fusion rate was $85.2 \%$ among 182 patients who were implanted with SAEFC, and ongoing loss of disc space height and lumbar lordosis contributed the operation of SAEFC being an inappropriate choice for patients with lumbar disc herniation. However, Neely et al. [18] reported that the SAEFC operation, in 470 patients, retained the stability and physiological lordosis by implanting two SAEFCs at each segment. Although a lower rate of subsidence was observed by the SAEFC approach, this method may cause greater trauma. 

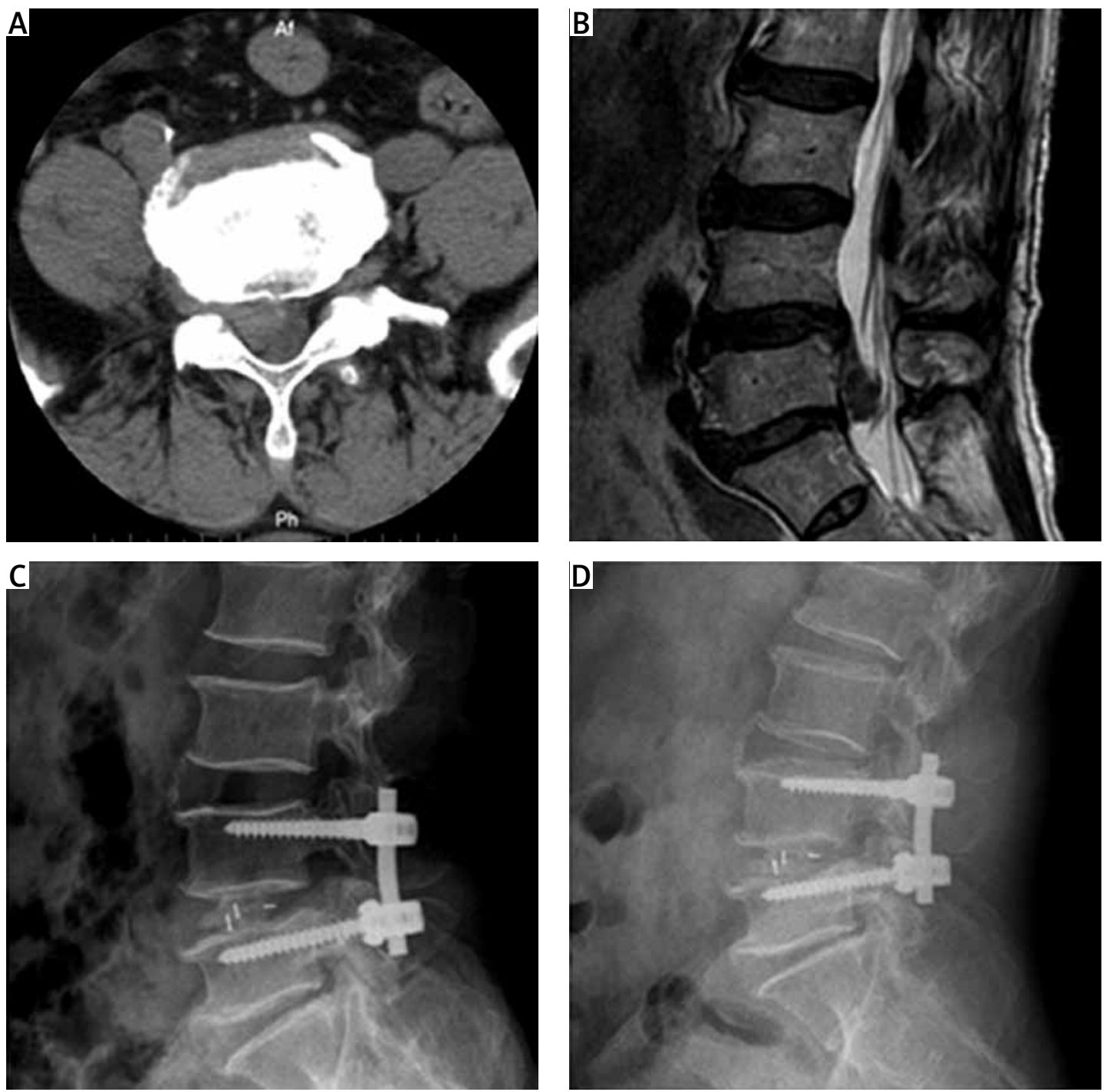

Figure 2. Pre- and post-operative images of a patient who received UPSFC. A - The preoperative image of CT scan for huge disc herniation at right side. B - Preoperative T2 WI MRI scanning for protrusion of intervertebral disc at L5/S1. C - X-ray image of lateral projection detected 1 week after operation. No alteration in the position of nail stick and cage was detected. D - X-ray image of lateral projection detected 1 year after operation

In this study, we selected patients with unilateral lumbar disc herniation and excluded bilateral disc herniation and lumbar spinal stenosis. For these patients, bilateral open-window decompression and even total laminectomy are often required. Under this condition, the stability of the lumbar spine is severely damaged. Lumbar fusion usually requires bilateral pedicle fixation and intervertebral fusion, which may not be suitable for both UPSFC and SAEFC. In some cases, decompression will inevitably destroy the stability of the structure of one side of the small joint, which can still be treated with UPSFEC, but it is not suitable for SAEFC. In order to compare the two techniques under the same conditions, the patients with unilateral lumbar disc herniation suitable for the two techniques were selected.

The original structures and biological stability of lumbar vertebra were well protected while carry- ing out these two operations. The stability of the zygapophyseal joint and integrity of the endplate cortex were well maintained. Damage to the zygapophyseal joint may influence its stability, which is unfavourable for fusion. Hence, no more than half of the zygapophyseal joints were removed. And patients with foraminal or extraforaminal disc herniation were excluded from this study because the zygapophyseal joint may be damaged during the decompression process. Patients with osteoporosis were also been eliminated from this research because osteoporosis in elderly patients can influence the stability of the zygapophyseal joint.

There are some limitations to our study: (1) The small sample size in this retrospective research may have influenced the results to some extent. (2) The follow-up period was too short to accurately evaluate long-term efficacy. There- 
fore, prospective studies with larger numbers of samples are warranted to further evaluate and certify the therapeutic effect of UPSFC and SAEFC. (3) Our study only included patients who suffered from unilateral disc herniation of a single segment, and the effect of UPSFC on other patients who are diagnosed with disc herniation of double- or multi-segments should be further investigated.

In conclusion, both SAEFC and UPSFC are effective techniques. UPSFC may be a better choice for patients with lumbar disc herniation and unilateral limb symptoms of the nerve root, in view of the advantages of better low back pain relief and low subsidence rate.

\section{Acknowledgments}

Informed consent was obtained from all subjects, and the study was approved by the Medical Ethics Committee of Zhoukou City Central Hospital (No. 2011012). Informed consent was obtained from all patients.

The authors acknowledge all colleagues who provided care to the patients.

\section{Conflict of interest}

The authors declare no conflict of interest.

\section{References}

1. Suk KS, Lee HM, Moon SH, Kim NH. Recurrent lumbar disc herniation: results of operative management. Spine (Phila Pa 1976) 2001; 26: 672-6.

2. Kim KT, Park SW, Kim YB. Disc height and segmental motion as risk factors for recurrent lumbar disc herniation. Spine (Phila Pa 1976) 2009; 34: 2674-8.

3. Hoogland T, van den Brekel-Dijkstra K, Schubert $M$, Miklitz B. Endoscopic transforaminal discectomy for recurrent lumbar disc herniation: a prospective, cohort evaluation of 262 consecutive cases. Spine (Phila Pa 1976) 2008; 33: 973-8.

4. Groff MW, Dailey AT, Ghogawala Z, et al. Guideline update for the performance of fusion procedures for degenerative disease of the lumbar spine. Part 12: pedicle screw fixation as an adjunct to posterolateral fusion. J Neurosurg Spine 2014; 21: 75-8.

5. Noshchenko A, Hoffecker L, Lindley EM, et al. Perioperative and long-term clinical outcomes for bone morphogenetic protein versus iliac crest bone graft for lumbar fusion in degenerative disk disease: systematic review with meta-analysis. J Spinal Disord Tech 2014; 27: 117-35.

6. Choi JY, Sung KH. Subsidence after anterior lumbar interbody fusion using paired stand-alone rectangular cages. Eur Spine J 2006; 15: 16-22.

7. Fogel GR, Toohey JS, Neidre A, Brantigan JW. Is one cage enough in posterior lumbar interbody fusion: a comparison of unilateral single cage interbody fusion to bilateral cages. J Spinal Disord Tech 2007; 20: 60-5.

8. Li X, Lv C, Yan T. Unilateral versus bilateral pedicle screw fixation for degenerative lumbar diseases: a meta-analysis of 10 randomized controlled trials. Med Sci Monit 2015; 21: 782-90.
9. Xiao SW, Jiang H, Yang LJ, Xiao ZM. Comparison of unilateral versus bilateral pedicle screw fixation with cage fusion in degenerative lumbar diseases: a meta-analysis. Eur Spine J 2015; 24: 764-74.

10. Rosenberg WS, Mummaneni PV. Transforaminal lumbar interbody fusion: technique, complications, and early results. Neurosurgery 2001; 48: 569-74.

11. Duncan JW, Bailey RA. An analysis of fusion cage migration in unilateral and bilateral fixation with transforaminal lumbar interbody fusion. Eur Spine J 2013; 22 439-45.

12. Lee JC, Kim Y, Soh JW, Shin BJ. Risk factors of adjacent segment disease requiring surgery after lumbar spinal fusion: comparison of posterior lumbar interbody fusion and posterolateral fusion. Spine (Phila Pa 1976) 2014; 39: E339-45.

13. Park P, Garton HJ, Gala VC, Hoff JT, McGillicuddy JE. Adjacent segment disease after lumbar or lumbosacral fusion: review of the literature. Spine (Phila Pa 1976) 2004; 29: 1938-44.

14. Phan K, Leung V, Scherman DB, Tan AR, Rao PJ, Mobbs RJ. Bilateral versus unilateral instrumentation in spinal surgery: systematic review and trial sequential analysis of prospective studies. J Clin Neurosci 2016; 30: 15-23.

15. Molinari RW, Saleh A, Molinari R Jr, Hermsmeyer J, Dettori JR. Unilateral versus bilateral instrumentation in spinal surgery: a systematic review. Global Spine J 2015; 5: 185-94.

16. Emstad E, Del Monaco DC, Fielding LC, Block JE. The VariLift((R)) Interbody Fusion System: expandable, standalone interbody fusion. Med Devices (Auckl) 2015; 8 : 219-30.

17. Shah RR, Mohammed S, Saifuddin A, Taylor BA. Comparison of plain radiographs with $C T$ scan to evaluate interbody fusion following the use of titanium interbody cages and transpedicular instrumentation. Eur Spine J 2003; 12: 378-85

18. Neely WF, Fichtel F, Del Monaco DC, Block JE. Treatment of symptomatic lumbar disc degeneration with the VariLift-L Interbody Fusion System: retrospective review of 470 cases. Int J Spine Surg 2016; 10: 15.

19. Xin Z, Li W. Unilateral versus bilateral pedicle screw fixation in short-segment lumbar spinal fusion: a metaanalysis of randomised controlled trials. Int Orthop 2016; 40: 355-64.

20. Kim KH, Park JY, Chin DK. Fusion criteria for posterior lumbar interbody fusion with intervertebral cages: the significance of traction spur. J Korean Neurosurg Soc 2009; 46: 328-32.

21. Park JH, Roh SW. Long-term clinical and radiological outcomes following stand-alone PLIF surgery using expandable cylindrical threaded cages in patients with degenerative lumbar spine disease. Acta Neurochir (Wien) 2011; 153: 1409-16.

22. Kim Y. Prediction of mechanical behaviors at interfaces between bone and two interbody cages of lumbar spine segments. Spine (Phila Pa 1976) 2001; 26: 1437-42.

23. Fuji T, Oda T, Kato Y, Fujita S, Tanaka M. Posterior lumbar interbody fusion using titanium cylindrical threaded cages: is optimal interbody fusion possible without other instrumentation? J Orthop Sci 2003; 8: 142-7. 